\title{
Free Signal Management in Cities Using Density and Average Speed
}

\author{
JAN MRAZEK \\ Department of Security Engineering \\ Tomas Bata University in Zlin, Faculty of Applied Informatics \\ Nad Stranemi 4511, Zlin, 76001 \\ CZECH REPUBLIC \\ jmrazek@utb.cz
}

\begin{abstract}
The article focuses on the issue of congested roads in cities. The problems of heavy traffic both large and smaller towns. Some towns in the Czech Republic do not have built bypasses and so there is a heavy traffic congestion at favorite times. Road transport has become one of the most popular forms of transport. This trend is unlikely to change in the near future. The current market offers dynamic traffic management but not with the appropriate effect. Our proposed method belongs to dynamic traffic control based on real traffic control. The proposed method works in real time with the light signaling device behind the monitoring sections. The function of the proposed method is to extend the free passage of the light-signaling device.
\end{abstract}

Key-Words: - Traffic, Traffic control, Traffic lights, Crisis management, Crisis Situation, Transport, Dynamic control, Clear Air.

Received: October 4, 2019. Revised: April 4, 2020. Accepted: April 28, 2020. Published: April 30, 2020.

\section{Introduction}

Heavy traffic in the city is a worldwide topical issue. Most countries are trying to solve this problem in their way. Germany, for example, by prohibiting cars from entering cities without meeting the EURO 5 emission limit. There is currently no methodology to propose dynamic traffic management. To prevent excessive saturation of roads in cities or city centers, dynamic traffic management must take place. Dynamic traffic management should work in real-time to avoid congestion and ensure throughput through the city without large columns. Over time, we know of places where they are fine and often cannot be prevented. However, these situations can occur for several reasons. The main causes are dense traffic flows flowing into cities for commuting to work or just passing through the city due to the absence of a bypass around the cit, [1], [2. ]Urban traffic management systems are numerous. The current offer on the market shows mainly dynamic systems. These systems should work with data in real-time. Just like any system has its advantages, these include disadvantages. The current market offer is very rich and can create a smart city in the form of smart cities. Dynamic control is currently limited to the current intersection where the intelligent control system is applied. The question is, however, whether it can be taken up a level.
Our proposed method is based on identifying points that are central to this system. Passage cameras will be built at selected locations to serve dynamic real-time traffic control. These places should be on the outskirts of towns or on the main arteries that bring the largest number of vehicles to towns. The method should work with the input data that we get from the transit cameras at the edges of the monitored city. Based on the information we work with and modeling, it should be possible to ensure smooth operation. The smooth operation should at the same time ensure increased safety and better air, because the means of transport often do not stand in columns. [3] The conclusion of the article should describe how the proposed method should work in practice. How this should guarantee the improvement of urban air while increasing safety. The use of the proposed method should ensure smooth operation and thus minimize the risk of traffic accidents. Traffic accidents are evaluated annually, these are current values that assess safety. As a result of increased safety, the number of road accidents must also be minimized. The aim is to manage the requirements from the perspective of vision 0 that governs Europe as a whole. The proposed system should support this vision in order to achieve it. 


\section{Current System in Practice for Control of Signals of Light Signal ling} Equipment

Technology development is moving very fast. This also leads to new systems, which are gradually trying to assert themselves in transport. The current offer on the market for the control of light signaling devices is currently sufficient. With current systems, it is possible to increase safety and ensure fluidity, while improving the city's air quality, [6], [7] .

Current systems in practice are often referred to as dynamic systems. However, this designation can be understood as being largely limited by the distance or maximum range at which they work. We will now become more familiar with the offer on the market [7], [8].

\subsection{Fixed Signaling Plane}

The fixed signaling device is the only representative of the static control of the light signaling device. From the point of view of developing technologies in a rocket-like way, however, the present time cannot replace this type of control. It can be stated that this would be an unnecessary process when this device has been operating in our country for many decades and is sufficient in many places.

\subsection{Variables Signaling Plane}

The function of variable signaling plans is based on defining the most popular times to increase the intervals at the places where constipation occurs. The most popular times can also be described as peak hours. Most often it is morning hours from 7 am to $8 \mathrm{pm}$ and afternoon from $2 \mathrm{pm}$ to $4 \mathrm{pm}$. These are intervals when we travel to work or take our children to school. Variable signaling plans can respond to these onslaughts based on predefined conditions by how much the green interval is extended.

\subsection{Video-detection}

Video-detection is an element that makes it possible to dynamically control the intersection where light signaling devices are installed. It is currently the most modern dynamic system. Video-detection is very effective and with various settings, it is possible to create very effective control of intersections. The big disadvantage, which we consider to be very influencing the dynamics of the control, is the distance that the video-detector is limited. This is the distance that this system can work.

\subsection{Detection of Induction Loops}

Induction loops are currently the most popular element used to control signals on light signaling devices. Certainly, we can classify them as dynamic elements. They open different directions or extend intervals as they pass. However, in heavy traffic, their dynamic control capability is limited by the distance from which they are installed. The big disadvantage of this signal is its implementation in the road. This causes considerable interference and limitations in case of its failure. After analyzing the available systems for dynamic control of the light signaling device, it can be stated that there are systems that can be applied and referred to as dynamic. Current systems limiting the distance at which they can work is predominantly limiting. The essence of smart cities is the cooperation of all elements together. For this reason, we are proposing a method that should be easier to create a smart city.

\section{Dynamic Operation Control Design}

The proposed dynamic control of the intervals of the light signaling device can be realized by means of transit cameras. These cameras are most often used for truck weighing or pass-through cameras for speed measurement. We chose the city of Zlín as an example. The city is partly specific because the bypass around the city is not built and therefore all traffic passes directly through the city. In Figure 1 you can see an example of control points where it would be appropriate to apply our proposed method. These points can be characterized as entry routes to the city, where most of the means of transport arrive and thus the city is most burdened. This place is called checkpoints and should be located at a sufficient distance from the city center. [3],[4],[5]

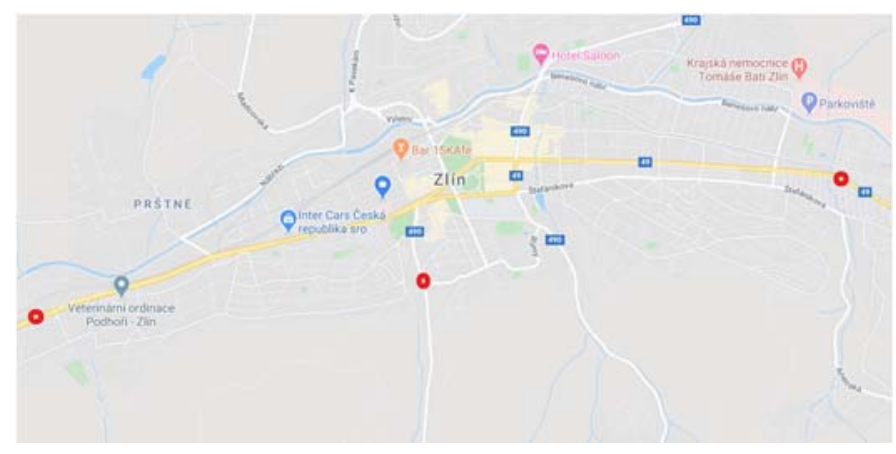

Fig. 1. Checkpoints in the city of Zlin.

In the picture, we can see the red dots. These points indicate checkpoints that should be used to retrieve data. Using real-time measured data, the control systems would then instruct all traffic lights in the measured section in the direction of the city center. 
Figure 2, which you can see below, is a real example of measured data. These data show us the traffic density, average speed and time at which the measurement took place.

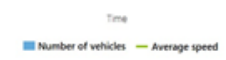

Fig. 2. Checkpoints in the city of Zlin.

Now let's get to know the different phases that should be set according to the needs of the specific city in which the method will be applied.

\subsection{Phase 1}

Phase 1 is referred to as an economically undemanding operation. It is a section where the traffic density is very low and the average speed on the monitored section is constant. At this time, the traffic lights are turned off. This situation occurs mostly on weekends holidays or evenings. These are intervals in which energy is saved because of the very low probability of a crisis situation. This is shown in the following figure with a red line.

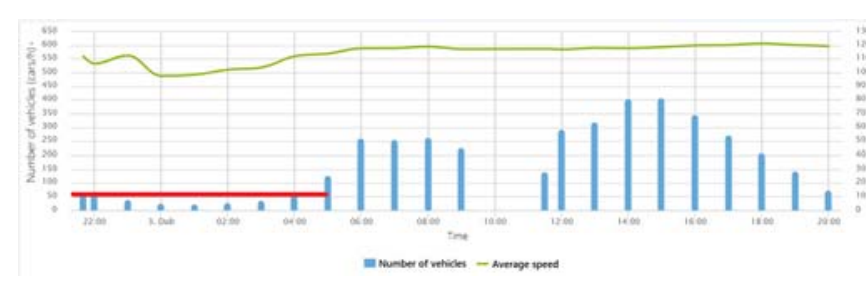

Fig. 3. Phase 1 in the city of Zlin

The red line in the picture shows us the time period during which the light signaling device should be switched off. This rule occurs only when the last hour has a low traffic density and current after 20 minutes does not show any major or sharp increase. We see an increase above a certain number of automobile at the end of the line. This is referred to as phase 2 .

\subsection{Phase 2}

In the next figure, a red dot and a label show Phase 2.

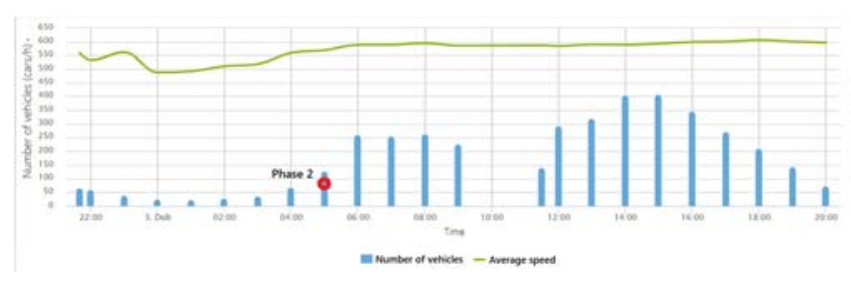

Fig. 4. Phase 2 in the city of Zlin

In Phase 2, the defined number of cars is exceeded and the light signaling devices are activated. This condition triggers traffic lights and at the same time sets the normal interval. The normal interval is set until the traffic density reaches the next phase.

\subsection{Phase 3}

In the picture below, you can see in advance whenever you want to move phase 2 to phase 3 .

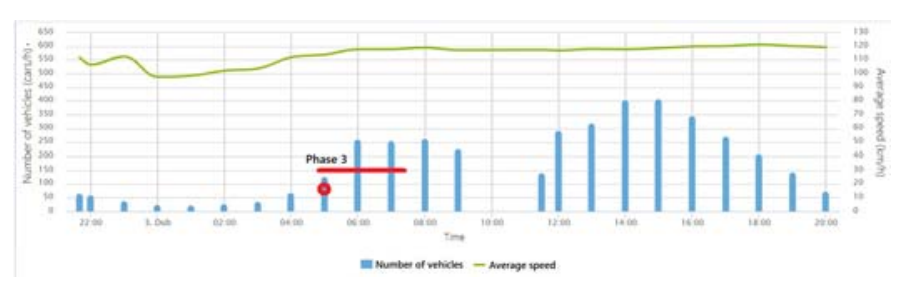

Fig. 5. Phase 3 in the city of Zlin

The difference between phase 2 and phase 3 is only in the length of the off signal on the light-signaling device. If the traffic density increases and exceeds from phase 2 to phase 3 , the "free pass" signal should be extended by 15 seconds or a pre-defined time. This time can be defined in advance and should be set taking into account the number of lanes in the monitored direction and the average speed.

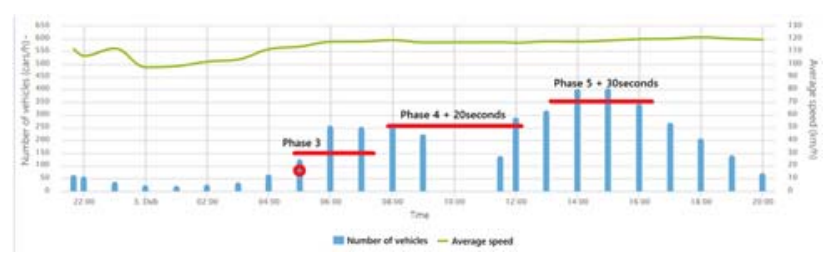

Fig. 6. Phase 4 to Phase 5 in the city of Zlin

In Figure 6 we can see other phases that can be programmed differently according to the needs of the particular method. With very heavy traffic, the interval can be extended by 5 minutes so that the method achieves the effect of healthier air, reduced emissions and energy savings. 
Reduction of emissions is ensured in cases when heavy traffic passes through the city at longer intervals so that no splitting and frequent braking and starting of the car occur.

The energy saving should be predominantly at night, when the traffic is very low and therefore the signaling device should be switched off. Switching to normal traffic occurs when Phase 1 is reached and therefore road safety is ensured.

\subsection{Phase X}

Phase $\mathrm{X}$ presents us with very extreme traffic density that will occur in the event of an obstacle and will thus significantly reduce the average speed in the monitored section. This stage may present a planned event, eg End of the concert in the town, etc. This phase is currently only present another possibility to use the proposed method. This situation is illustrated in Figure 7. This situation should only be applied in exceptional cases in order to speed up the release of traffic. This loosening should be in the vicinity of the event where the event took place to reduce the risk of column formation or traffic accidents.

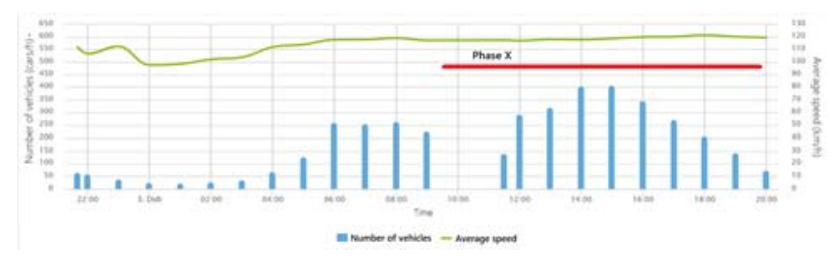

Fig. 6. Phase $\mathrm{X}$ - extraordinary event.

The extraordinary event, or phase $\mathrm{X}$, should serve to free up the venue of the event.

\section{Conclusion}

Concurrent times allow us to hurry life. We teach this speech to our children from a very young age. We will announce this opportunity too late. Children adapt to our lifestyles sometimes sooner. This manifestation mirrors and changes with time.

A busy time tries us even when driving a car. When moving on a public road, we are surrounded by people, construction and traffic signs, we receive a light signal. A large number of drivers are brought up in driving schools for driving employing a light signaling device. Weighting, whenever an intersection policeman is most of the drivers confused and ignorant. This manifestation creates additional stress among drivers, which may increase to increase the number of road accidents that endanger the health behind the wheel. Traffic density indicates an increase in traffic. These data are presented from both city and transit cameras on motorways. This trend is appropriate and a greater number of transport benefits will not be hostile to it. Pushing cars out of city centers is just a common solution. Based on such reactions, the cause is not solved, but the delay is delayed until the problem can outgrow and lead to fatal consequences. We already have dynamic elements that work in realtime based on traffic density. The development of new intelligent systems can be observed every year. These systems help us, but for dynamic traffic control, it is necessary to work with input data to have a robotic control based on artificial intelligence. Artificial intelligence should be able to expect data from past periods and perceive them as real data redundancy for more accurate and efficient management. The proposed system works in realtime. Its advantage is working with data in recent hours and can free crowded streets in city centers to make traffic smoother, safer and improve the air we breathe. The next step should be testing the proposed method under real conditions. Application of the system and testing can provide us with data that will be able to evaluate subsequently whether the proposed method and method of solution meets the requirements for traffic management.

\section{Acknowledgment:}

This project is realized as the research with doctoral student and it is the basic input for next research, which we will develop in next term. It was realized with support of the university. This work was supported by Internal Grant Agency of Tomas Bata University under the project No. IGA/FAI/2020/002.

References:

[1] BLACK, John. Urban transport planning: Theory and practice. Routledge, 2018.

[2] EHSANI, Mehrsa; AHMADI, Abbas; FADAI, Dawud. Modeling of vehicle fuel consumption and carbon dioxide emission in road transport. Renewable and sustainable energy reviews, 2016, 53: 1638-1648.

[3] GABRIEL, Cynthia Ann; SHAO, Bill. Dynamic traffic management in a data center. U.S. Patent No 9,749,241, 2017.

[4] Garza-Reyes, J. A., Tangkeow, S., Kumar, V. and Nadeem, S. P. and NA (2018) Lean manufacturing adoption in the transport and logistics sector of Thailand - An exploratory study. Proceedings of the International Conference on Industrial Engineering and 
Operations Management Bandung, Indonesia, March 6-8, 2018, ID 45. pp. 104-115. ISSN 2169-8767 I. S. Jacobs and C. P. Bean, "Fine particles, thin films and exchange anisotropy," in Magnetism, vol. III, G. T. Rado and H. Suhl, Eds. New York: Academic, 1963, pp. 271-350.

[5] CHAI, Huajun, et al. Dynamic traffic routing in a network with adaptive signal control. Transportation Research Part C: Emerging Technologies, 2017, 85: 64-85.

[6] MRAZEK, Jan, DURICOVA, Lucia \& HROMADA, Martin. „The Proposal of Software for Transport Infrastructure Management". In IISA 2017 - The 8th International Conference on Information, Intelligence, Systems and Applications, Larnaca, Cyprus, 28. 8. - 30. 8. 2017, ISBN 978-1-5090-342-1, 2017.

[7] MRAZEK, Jan, DURICOVA, Lucia \& HROMADA, Martin. The Proposal of Evaluation Criteria for Recoverability of Road Transport. Safety and Reliability - Theory and Applications - Čepins \& Briš (Eds), 2017. Taylor \& Francis Group, London. ISBN 978-1138-62937-0.

[8] WANG, Yi, et al. Dynamic traffic assignment: A review of the methodological advances for environmentally sustainable road transportation applications. Transportation Research Part B: Methodological, 2018, 111: 370-394.

[9] WANG, Yi, et al. Dynamic traffic assignment: A review of the methodological advances for environmentally sustainable road transportation applications. Transportation Research Part B: Methodological, 2018, 111: 370-394.

[10] ZHANG, Ying. Method and system to dynamically collect statistics of traffic flows in a software-defined networking (sdn) system. U.S. Patent Application No 14/462,444, 2016.

[11] ZHANG, Linfeng, et al. Deep potential molecular dynamics: a scalable model with the accuracy of quantum mechanics. Physical review letters, 2018, 120.14: 143001. 